\title{
A SUBCATEGORY OF TOP
}

\author{
ALAN DOW AND STEPHEN WATSON
}

\begin{abstract}
We consider the smallest class of topological spaces which contains the converging sequence and which is closed under the operations of taking arbitrary sums, quotients and finite products. We show that if there is a model of set-theory in which there is a measurable cardinal then there is a model in which this class does not contain all topological spaces. In addition, we prove that it is consistent that this class does contain all topological spaces-in fact much more, a large cardinal is needed to produce a model of set theory in which this class is proper.
\end{abstract}

\section{INTRODUCTION}

In [7], M. Husek asks if there can be a proper subclass of TOP which is closed under the operations of sum, quotient and arbitrary products. The question is asked again in Herrlich's survey [6]. We show that it is consistent that we do obtain all spaces. In fact, by utilizing Todorcevic's results on raising tightness in finite products we obtain that the consistency of not getting all spaces implies the consistency of there being a weakly compact cardinal. In addition, the proof even shows that we (consistently) obtain all spaces in the smallest class which is closed under sums, quotients and only finite products (however in the case of finite products we must start with the converging sequence to get started). We then show that it is consistent that finite products are not enough to raise tightness arbitrarily high (assuming the consistency of a strongly compact cardinal). In addition, if there are measurable cardinals, then the class which is generated by taking sums, quotients and finite products is a proper subclass of TOP.

Let $\mathfrak{C}$ denote the smallest subcategory of TOP which contains the converging sequence $\omega+1$ and which is closed under sums, quotients and finite products. If we assume, in addition, that $\mathfrak{C}$ is closed under arbitrary products then there is no need to assume that $\omega+1$ is in $\mathfrak{C}$. This is because the product of empty sets is a set containing the singleton element, the empty function, a sum of two of these is the usual two point space. Finally, the converging sequence is a quotient of $2^{\omega}$.

In the first section we will see the effect of sums and quotients on $\mathfrak{C}$. We show that if $\mathfrak{C}$ contains, for each infinite cardinal $\kappa$, a space with an open discrete subset of size $\kappa$ which witnesses tightness $\kappa$ in a strong sense, then $\mathfrak{C}$ is all of

Received by the editors July 20, 1990 and, in revised form, March 8, 1991.

1980 Mathematics Subject Classification (1985 Revision). Primary 54B10; Secondary 54B15, $54 \mathrm{~A} 25,54 \mathrm{~A} 35$. 
TOP. In the second section we will show the consistency of $\mathfrak{C}$ equaling TOP by giving a proof of Todorcevic's result concerning finite powers of sequential spaces. In the third section we will assume the existence of a measurable cardinal and prove the consistency of $\mathfrak{C}$ being a proper subclass of TOP. However in doing so we actually produce two strikingly different models of this statement. In the first case, it is not very surprising that the existence of a measurable cardinal, $\theta$, (in a model) implies that some very large spaces (of cardinality $\theta$ ) are not in $\mathfrak{C}$. It will follow from our results in the earlier sections, that in such a model, it may still be the case that $\mathfrak{C}$ includes every space with cardinality less than the first weakly compact cardinal. However we also show that if $\theta$ is real-valued measurable but not measurable then there are countable spaces which are not members of $\mathfrak{C}$. We also relate these results to the tightness of spaces in the class $\mathfrak{C}$ and, with the assumption of the existence of a strongly compact cardinal, we show that the tightness function is bounded in the class $\mathfrak{C}$. In the final section we pose some open questions.

\section{SUMS AND QUOTIENTS}

For an indexed family of spaces $\left\{X_{i}: i \in I\right\}, \sum_{i \in I} X_{i}$ denotes the topological sum of the family. That is, the topological sum has base set $\bigcup X_{i} \times\{i\}$ and is topologized as follows. For each $i \in I, X_{i} \times\{i\}$ is clopen and $U \times\{i\}$ is open if and only if $U \subset X_{i}$ is open. A space $Y$ is a quotient of a space $X$ if there is a quotient map $f: X \mapsto Y$. The function $f: X \mapsto Y$ is a quotient map if it is onto and if $Y$ has the finest topology which makes $f$ continuous. There are useful equivalent criteria.

Proposition 2.1 [3, 2.4.3]. For a mapping $f$ of a topological space $X$ onto a space $Y$ the following are equivalent:

(1) The mapping $f$ is a quotient map.

(2) The set $f^{-1}(U)$ is open in $X$ iff $U$ is open in $Y$.

(3) The set $f^{-1}(F)$ is closed in $X$ iff $F$ is closed in $Y$.

Lemma 2.2. Let $Y$ be a space with unique nonisolated point $q$, and let $X$ be any space. Let $P$ be a subset of $X$ which contains all of the nonisolated points of $X$. Suppose that there are subsets $\left\{O_{y}: y \in Y-\{q\}\right\}$ of $X-P$ such that, for each $S \subset Y-\{q\}$,

$$
S \text { is closed (in } Y \text { ) iff } \bigcup_{y \in S} O_{y} \text { is closed (in } X \text { ) }
$$

then $Y$ is a quotient of a sum of copies of $X$.

Proof. We give $X \times \mathscr{P}(Y-\{q\})$ the "sum" topology. Assume that $Y$ has an associated well ordering, that $q$ is the largest element in the well ordering, and let $O_{q}=Y$. Define the function $f$ from $X \times \mathscr{P}(Y-\{q\})$ to $Y$ as follows:

$$
f(x, S)=\min \left\{y \in S \cup\{q\}: x \in O_{y}\right\} .
$$

Note that if $p \in P$, then $f(p, S)=q$ for any $S \subset Y-\{q\}$. Let $H$ be an arbitrary subset of $Y$. We will show that $H$ is closed in $Y$ if and only if $f^{-1}(H)$ is closed in $X$. First of all, if $q \in H$ then both $H$ and $f^{-1}(H)$ are closed. Next suppose that $H \subset Y-\{q\}$ is closed. Let $S \subset Y-\{q\}$ be arbitrary. If $f(x, s) \in H$, then $x \in \bigcup_{y \in S \cap H} O_{y}$. By our hypothesis, this set is 
closed since $S \cap H$ is closed. This verifies that $f^{-1}(H) \cap X \times\{S\}$ is closed. Since $S$ was arbitrary and $X \times \mathscr{P}(Y-\{q\})$ has the sum topology, $f^{-1}(H)$ is closed. Finally, assume that $q \notin H$ and that $f^{-1}(H)$ is closed. Since $f^{-1}(H)$ intersected with $X \times\{H\}$ is closed, we have that $\bigcup_{y \in H} O_{y}$ is closed. Again, by our hypothesis, $H$ is closed.

For a cardinal $\theta$, let $\theta \oplus 1$ denote the space with base set $\theta+1$ and in which each $\alpha<\theta$ is isolated and the set of infinite final segments form a base for $\theta$. Let us say that a subset $S$ of a space $X$ witnesses tightness $\theta$ if $S$ has cardinality $\theta, S$ has limit point $x$ but $x$ is not a limit of any subset of $S$ of cardinality less than $\theta$. A set $S$ witnesses tightness precisely $\theta$ if $S$ witnesses tightness $\theta$ but no subset of $S$ of smaller cardinality has a limit point. We will say that a space witnesses tightness (precisely) $\theta$ if it has some subset which witnesses tightness (precisely) $\theta$.

Lemma 2.3. Suppose that $\theta$ is a cardinal and that $\mathfrak{C}$ contains some space $X$ which has a unique nonisolated point and which witnesses tightness $\theta$ then $\mathfrak{C}$ contains the space $\theta \oplus 1$.

Proof. We will simply use Lemma 2.2. Let $p$ be the nonisolated point of $X$ and let $\left\{x_{\alpha}: \alpha<\theta\right\}$ be the subset which witnesses tightness $\theta$. For each $\alpha<\theta$, let $O_{\alpha}=\left\{x_{\beta}: \beta<\alpha\right\}$. It is easily seen that for any $S \subset \theta, p$ is a limit point of the union of the family $\left\{O_{\alpha}: \alpha \in S\right\}$ if and only if $S$ is cofinal in $\theta$.

Lemma 2.4. Let $\theta$ be an infinite cardinal and assume that for every regular cardinal $\lambda \leq \theta$, the space $\lambda \oplus 1$ is in $\mathfrak{C}$. Then $\mathfrak{C}$ contains every space $X$ which has character at most $\theta$.

Proof. Let us begin by defining a class of elementary spaces. For a cardinal $\theta$, let $\mathfrak{E}_{\theta}$ denote the class of all spaces $Y$ with the following properties: $Y$ has a unique nonisolated point $q, q$ has a neighborhood base of cardinality at most $\theta$, and no subspace of $Y$ in which $q$ is nonisolated has smaller character or cardinality. Note, though, that the cardinality of $Y$ can be less than the character.

We shall prove, by induction on $\theta$, that if $\lambda \oplus 1 \in \mathfrak{C}$ for each regular $\lambda \leq \theta$, then $\mathfrak{C}$ contains $\mathfrak{E}_{\theta}$. The lemma can be deduced from this fact and since this will be required in the proof we do this first. Indeed, suppose that $X$ is a space with character at most $\theta$ and suppose that $\mathfrak{C}$ contains $\mathfrak{E}_{\theta}$. We want to express $X$ as a quotient of a sum of spaces in $\mathfrak{E}_{\theta}$. For each $x \in X$, let $X_{x}$ denote the space where $x$ retains its neighborhood base from $X$ and every other point is isolated. The mapping $f$ from $\sum_{y \in X} X_{y}$ to $X$ defined by $f(x, y)=x$ for each $x \in X_{y}$ is a quotient mapping. Therefore, if $X_{x} \in \mathfrak{C}$ for each $x \in X$, then $X \in \mathfrak{C}$. Similarly, it is clear that $X_{x}$ is a quotient, via the obvious map, of the sum of all subspaces of $X_{X}$ which are in $\mathfrak{E}_{\theta}$.

Assume that $\lambda \oplus 1 \in \mathfrak{C}$ for all regular $\lambda \leq \theta$. Let $Y$ be an arbitrary element of $\mathfrak{E}_{\theta}$ and assume by induction that $\mathfrak{E}_{\lambda} \subset \mathfrak{C}$ for all $\lambda<\theta$. Let $\mathfrak{U}$ be a neighborhood base for $q$ with cardinality $\theta$. Let $\left\{U_{\alpha}: \alpha<\theta\right\}$ enumerate $\mathfrak{U}$ so that each member appears cofinally often. For $\alpha<\theta$, let $\mathfrak{U}_{\alpha}$ denote the filter generated by $\left\{U_{\beta}: \beta<\alpha\right\}$. By inductive assumption and the discussion above, $\mathfrak{C}$ contains every space of character less than $\theta$. Therefore the space $Y_{\alpha}$ is in $\mathfrak{C}$, where $Y_{\alpha}$ is obtained by taking the filter $\mathfrak{U}_{\alpha}$ as a neighborhood for the point, $p_{\alpha}$, which is obtained by collapsing the set $\bigcap\left\{U: U \in \mathfrak{U}_{\alpha}\right\}$ to a 
point. Similarly, if $\theta$ is a singular cardinal, then $\mathfrak{C}$ contains $\theta \oplus 1$ since the character of this space is less than $\theta$. Let $Z$ be the quotient space obtained by collapsing all the nonisolated points of $\sum_{\alpha<\theta} Y_{\alpha}$ to a single point $p$. Let $X$ denote the product $Z \times(\theta \oplus 1)$. Note that isolated points of $X$ are of the form $((y, \beta), \gamma)$, where $y \in Y_{\beta}-\left\{p_{\beta}\right\}$ and the nonisolated points, $P$, are of three types: $p$ cross the isolated points of $\theta \oplus 1$, the isolated points of $Z$ cross the singleton $\theta$ and finally the point $(p, \theta)$. We will show that $Y$ is a quotient of $X$ (using 2.2) which will complete the proof.

For each $y \in Y-\{q\}$, let

$$
O_{y}=\left\{((y, \beta), \gamma): \gamma<\beta<\theta \text { and } y \notin U_{\gamma}\right\} .
$$

Let $((y, \beta), \gamma) \in O_{y}$. Since $\beta>\gamma$ and $y \notin U_{\gamma}$, we see that $y \in Y_{\beta}-\left\{p_{\beta}\right\}$. Therefore $O_{y}$ consists entirely of isolated points of $X$.

Claim. For each $\gamma<\theta$, no point of $Z \times\{\gamma\}$ is a limit point of the family $\left\{O_{y}: y \in Y-\{q\}\right\}$.

Indeed, for $\alpha \leq \gamma$ and $y \in Y-\{q\}, O_{y} \cap Y_{\alpha} \times\{\gamma\}$ is empty. For $\alpha>$ $\gamma,\left(U_{\gamma} \times\{\alpha\}\right) \times\{\gamma\}$ is a neighborhood of $p_{\alpha}$ which misses all of the $O_{y}$ 's. Therefore, there is a quotient neighborhood $W$ of $p$ so that $W \times\{\gamma\}$ is disjoint from every $O_{y}$.

Claim. For each $\alpha<\theta$ and $y \in Y_{\alpha}-\left\{p_{\alpha}\right\},((y, \alpha), \theta)$ is not a limit point of the family $\left\{O_{z}: z \in Y-\{q\}\right\}$.

Indeed, $(y, \alpha) \times(\alpha, \theta]$ is a neighborhood of $((y, \alpha), \theta)$ which does not intersect any $O_{y}$.

We finish the proof by proving the following fact which shows the applicability of Lemma 2.2.

Fact. For each $S \subset Y-\{q\}$,

$$
q \in \bar{S} \text { iff }(p, \theta) \text { is a limit point of } \bigcup_{y \in S} O_{y} .
$$

For each $\beta<\theta$, let us let $Z_{\beta}$ denote the subset $\left(Y_{\beta}-\left\{p_{\beta}\right\}\right) \times\{\beta\}$ of $Z$.

Suppose first that $S \subset Y-\{q\}$ and that $(p, \theta)$ is a limit of $\bigcup_{y \in S} O_{y}$. Let $\alpha<\theta$, we must show that $U_{\alpha} \cap S \neq \varnothing$. Let $W$ be the following neighborhood of $p$ (in $Z$ ): for each $\beta<\alpha, W \supset Z_{\beta}$, and, for each $\beta \geq \alpha, W \cap Z_{\beta}=$ $U_{\alpha}-\{q\} \times\{\beta\}$. Since $W \times(\alpha, \theta]$ is a neighborhood of $(p, \theta)$ it intersects $\bigcup_{y \in S} O_{y}$. Let $((y, \beta), \gamma)$ be a member of $O_{y} \cap W \times(\alpha, \theta)$ for some $y \in S$. Clearly then, $\gamma>\alpha$ and, from the definition of $O_{y}$, we deduce that $\beta>\alpha$. It then follows by the definition of $W$, that $y \in U_{\alpha}$, hence $U_{\alpha} \cap S \neq \varnothing$.

Now suppose that $S \subset Y$ and that $q \in \bar{S}$-hence, $U_{\alpha} \cap S \neq \varnothing$ for all $\alpha<\theta$. We must show that $(p, \theta)$ is a limit point of $\bigcup_{y \in S} O_{y}$. Let $W$ be a neighborhood of $p$ in $Z$. Choose a sequence $\left\{F_{\alpha}: \alpha \in \theta\right\}$ so that, for each $\alpha, F_{\alpha}$ is a finite subset of $\alpha$, and $W \supset \bigcap_{\xi \in F_{\alpha}}\left(U_{\xi}-\{q\}\right) \times\{\alpha\}$. Now suppose that $W \times(\lambda, \theta] \cap \bigcup_{y \in S} O_{y}$ is empty.

First we handle the case when $\theta$ is a regular cardinal. Since each $F_{\alpha}$ is contained in $\alpha$ we may use the pressing down lemma to obtain a cofinal set $T$ in $\theta$ and a finite $F \subset \theta$ so that $F_{\beta}=F$ for all $\beta \in T$. Now choose an $\alpha>\lambda$ so that $\left(S \cap \bigcap_{\xi \in F} U_{\xi}\right)-U_{\alpha}$ is not empty (if we could not, then the character of $q$ in the subspace $S \cap \bigcap_{\xi \in F} U_{\xi} \cup\{q\}$ would be less than $\theta$ ). Next choose 
$\beta \in T$ so that $\beta>\alpha$. Hence, for each $y \in\left(\bigcap_{\xi \in F} U_{\xi} \cap S\right)-U_{\alpha},((y, \beta), \alpha)$ is in $(W \times(\lambda, \theta]) \cap O_{y}$.

Now we assume that $\theta$ is singular. Choose an increasing cofinal sequence in $\theta,\left\{\alpha_{\beta}: \beta<\operatorname{cf}(\theta)\right\}$. For each $\beta<\operatorname{cf}(\theta)$, choose $s_{\beta} \in S \cap \bigcap_{\xi \in F_{\alpha \beta}} U_{\xi}$. If some initial segment of $\left\{s_{\beta}: \beta<\operatorname{cf}(\theta)\right\}$ has $q$ as a limit point then, by our assumption on minimal tightness, $|S|<\operatorname{cf}(\theta)$. Therefore, there is some $s$ so that $s=s_{\beta}$ for cofinally many $\beta$ in $\operatorname{cf}(\theta)$. On the other hand if every initial segment is closed, then some cofinal sequence does not have $q$ as a limit (otherwise the subspace would have character less than $\theta$ ). In either case, there is a cofinal set $I \subset \operatorname{cf}(\theta)$ so that $\left\{s_{\beta}: \beta \in I\right\}$ is closed. Since each neighborhood in the base is listed cofinally often, we may choose an $\alpha>\lambda$ so that $U_{\alpha} \cap\left\{s_{\beta}: \beta \in I\right\}$ is empty. Choose $\beta \in I$ so that $\alpha_{\beta}>\alpha$ and, as in the regular case, note that $\left(\left(s_{\beta}, \alpha_{\beta}\right), \alpha\right)$ is in $O_{s_{\beta}}$ and in $W \times(\lambda, \theta)$.

Now from the above lemmas we can deduce the following result.

Theorem 2.5. If, for every regular cardinal $\theta$, there is a space $X \in \mathfrak{C}$ which has a set of isolated points which witnesses tightness precisely $\theta$ then $\mathfrak{C}=$ TOP.

Proof. Let $X$ be a space which has a set $S$ of isolated points which witnesses tightness precisely $\theta$. Let $P=X \backslash S$. The quotient space obtained by collapsing $P$ to a point is a space which has a unique nonisolated point and which witnesses tightness $\theta$. By Lemma 2.3, the space $\theta \oplus 1$ is in $\mathfrak{C}$ for each regular cardinal $\theta$. Now we apply Lemma 2.4 .

Remark 2.6. For historical reasons let us remark that it can now be shown to follow from the assumption "GCH plus no inaccessible cardinals" that $\mathfrak{C}$ is all of TOP. That is, by induction, one can show, under these assumptions, that the hypotheses of Theorem 2.5 hold. Indeed, suppose that $\theta$ is regular such that $\lambda \oplus 1$ is in $\mathfrak{C}$ for each regular $\lambda<\theta$. By Lemma $2.4, \mathfrak{C}$ contains all quotients and sums of spaces with character less than $\theta$. Since there are no inaccessibles, there is a $\lambda$ such that $\theta=\lambda^{+}$. Malyhin [9], has shown that there are spaces $X$ and $Y$ with tightness $\lambda$ whose product witness tightness (precisely) greater than $\lambda$. However we require that the spaces $X$ and $Y$ are quotients of spaces known to be in $\mathfrak{C}$. This can be done by a modification of the construction by Arhangelskii in [1] (which in turn is based on Stone's construction [11]) which handles the case when $\lambda=\omega$. It is shown that $X \times Y$ witnesses tightness precisely $\kappa$ for some $\kappa$ with $\omega<\kappa \leq 2^{\omega}$ where $X$ is the quotient of countably many copies of $\omega \oplus 1$ and $Y$ is the quotient of $2^{\omega}$ copies of $\omega \oplus 1$ where in both cases all nonisolated points are identified to one point. When $\lambda$ is regular, we can replace $\omega$ everywhere by $\lambda$ in Arhangelskii's construction. In case $\lambda$ is singular, it is not difficult to generalize the idea as follow. Let $\kappa$ be the cofinality of $\lambda$ and let $\left\{\lambda_{\alpha}: \alpha<\kappa\right\}$ be an increasing sequence of regular cardinals cofinal in $\lambda$. For $X$, take the analogous quotient of the sum of all the spaces $\lambda_{\alpha} \oplus 1$ while for $Y$ take the quotient of $\left|\prod\left\{\lambda_{\alpha}: \alpha<\kappa\right\}\right|$-many copies of $\kappa \oplus 1$.

\section{Products}

For a set $I$, we let $F_{I, \omega}$ denote the Fréchet $I$-fan which is obtained by collapsing all the nonisolated points to a single point in the topological sum of $I$ copies of $\omega+1$. We shall represent these spaces as $\{\infty\} \cup I \times \omega$. Note 
that the neighborhood basis for $\{\infty\}$ can be parametrized by functions from $I$ to $\omega$ where for such a function, $h$, the neighborhood coded by $h$ is $\{\infty\} \cup$ $\{(i, n): h(i) \leq n\}$.

In this section we present, with Todorcevic's kind permission, the proof from [13], that, in the absence of large cardinals, the square of the Fréchet $\theta$-fan witnesses tightness precisely $\theta$, for every infinite regular cardinal $\theta$. Arhangelskii showed in [1], that the square of the $\mathfrak{c}$-fan has uncountable tightness.

Let $\theta$ be an uncountable regular cardinal. A sequence $\left\langle C_{\alpha}: \alpha\langle\theta\rangle\right.$ is a $\square(\theta)$-sequence if $C_{\alpha+1}=\{\alpha\}, C_{\alpha}$ is a closed and unbounded (cub) subset of $\alpha$ for $\alpha$ a limit, and $C_{\alpha}=C_{\beta} \cap \alpha$ for $\alpha$ a limit in $C_{\beta}$, but there is no cub $C$ in $\theta$ such that $C \cap \alpha=C_{\alpha}$ for all $\alpha$ which are limits in $C$. Note that for $\theta=\lambda^{+}$, this is a considerable weakening of the usual $\square_{\theta}$ of Jensen [8]. A result of Jensen (see [8 and 14,1.10]) says that if $\theta$ is not weakly compact in $L$ then $\square(\theta)$ holds. The $\square(\theta)$-sequence $\left\langle C_{\alpha}: \alpha<\theta\right\rangle$ determines a function $\rho_{2}:[\theta]^{2} \mapsto \omega$ recursively by

$$
\rho_{2}(\alpha, \beta)=\rho_{2}\left(\alpha, \min \left(C_{\beta}-\alpha\right)\right)+1,
$$

(where $\alpha<\beta$ ). In $[14,1.14]$, it is shown that the sequence of functions $\rho_{2}(\cdot, \alpha): \alpha \mapsto \omega$ satisfies certain nonextendibility and coherence properties. The only properties of this sequence used in [13] are contained in the following definition which is modelled after a concept introduced in [2].

Definition 3.1. Let $\theta$ be an ordinal. A family $\left\{f_{\alpha}: \alpha<\theta\right\}$ is called a monotone $\theta$-family (of functions) if, for each $\beta \leq \alpha<\theta, f_{\alpha} \in{ }^{\alpha} \omega$ and there is an $n_{\beta, \alpha} \in \omega$ such that $f_{\beta}(\gamma)<f_{\alpha}(\gamma)+n_{\beta, \alpha}$ for each $\gamma<\beta$. A monotone $\theta$-family $\left\{f_{\alpha}: \alpha<\theta\right\}$ is nonextendible if it does not extend to a monotone $\theta+1$-family.

Now we give the application to tightness in products.

Theorem 3.2 (Todorcevic). If $\theta$ is an infinite cardinal such that there is a nonextendible monotone $\theta$-family then the square of the space $F_{\theta, \omega}$ witnesses tightness precisely $\theta$.

Proof. Let $\left\{f_{\alpha}: \alpha<\theta\right\}$ be the nonextendible monotone family. For $\beta<\gamma<$ $\theta$, let $n_{\beta, \gamma}$ denote the integer as in the definition of monotone family. Define

$$
S=\left\{((\alpha, m),(\beta, k)): \alpha<\beta \text { and } m+k<f_{\beta}(\alpha)\right\} .
$$

We show that $S$ witnesses tightness precisely $\theta$.

Small subsets of $S$ are closed. Let $\gamma<\theta$ and define $h_{\gamma} \in{ }^{\theta} \omega$ by

$$
h_{\gamma}(\alpha)= \begin{cases}f_{\gamma}(\alpha)+n_{\alpha, \gamma}+1 & \text { if } \alpha<\gamma, \\ 0 & \text { if } \alpha \geq \gamma .\end{cases}
$$

We first show that the neighborhood of $(\infty, \infty)$ which is obtained by squaring the neighborhood coded by $h_{\gamma}$ does not contain any points in the set $S \cap(\gamma \times \omega)^{2}$. Assume that both $(\alpha, m)$ and $(\beta, k)$ are in the neighborhood coded by $h_{\gamma}$ and that $\alpha<\beta<\gamma$. It follows then that

$$
m \geq h_{\gamma}(\alpha)>f_{\gamma}(\alpha) \text { and } k \geq h_{\gamma}(\beta)>n_{\beta, \gamma} .
$$

Therefore $m+k>f_{\gamma}(\alpha)+n_{\beta, \gamma}>f_{\beta}(\alpha)$. Hence $((\alpha, m),(\beta, k))$ is not in $S$. 
$S$ is closed in $\left(F_{\theta, \omega}\right)^{2}-\{(\infty, \infty)\}$. The function $f_{\alpha}$ witnesses that $(\infty,(\alpha, n))$ is not a limit of $S$ and the function $g(\gamma)=f_{\gamma}(\alpha)+n_{\alpha, \gamma}$ witnesses that $((\alpha, n), \infty)$ is not a limit of $S$.

$S$ is not closed. Let $W$ be an arbitrary neighborhood of $(\infty, \infty)$ and let $h$ be a function which codes a neighborhood of $\infty$ whose square is contained in $W$. Since our monotone family is not extendible, there is an $\beta<\theta$ such that $f_{\beta}$ is not less than $h+n$ for any integer $n$. For this reason we can choose $\alpha<\beta$, so that $f_{\beta}(\alpha)>h(\alpha)+h(\beta)$. Therefore $((\alpha, h(\alpha)),(\beta, h(\beta)))$ is in $S$ and by the choice of $h$, it is in $W$.

This completes the proof.

Combined with Theorem 2.5 we obtain the following result.

Theorem 3.3. If $\theta$ is a cardinal such that $\square(\lambda)$ holds for all regular uncountable $\lambda<\theta$, then the class $\mathfrak{C}$ contains every space $X$ of character less than $\theta$.

Corollary 3.4. There is a model of set-theory in which $\mathfrak{C}$ is equal to TOP. In fact, the consistency of $\mathfrak{C}$ not equal to TOP, implies the consistency of the existence of a weakly compact cardinal.

\section{ReAl-VALUED MEASURABLE CARDINALS}

Definitions. A measure is a finitely additive $[0,1]$-valued function, $\mu$, defined on an algebra of sets $B \subset \mathscr{P}(I)$, for some set $I$, such that $\mu(\{i\})=0$ for all $i \in I$ and $\mu(I)>0$. A two-valued measure has the obvious meaning. We let $\operatorname{add}(\mu)$ denote the minimum cardinal $\lambda$ such that there are pairwise disjoint sets $A_{\alpha} \in B, \alpha<\lambda$, such that $\sum_{\alpha<\lambda} \mu\left(A_{\alpha}\right)$ is not equal to $\mu\left(\bigcup_{\alpha<\lambda}\left(A_{\alpha}\right)\right)$. A measure, $\mu$, is $\kappa$-additive if $\kappa \leq \operatorname{add}(\mu)$. We let unif $(\mu)$ denote the minimum cardinality of a set $X \subset I$ such that $\mu(X) \neq 0$.

A cardinal $\kappa$ is measurable if $\kappa>\omega$ and there is a $\kappa$-additive two-valued measure defined on all of $\mathscr{P}(\kappa)$.

A cardinal $\kappa$ is strongly compact if, for every set $I$ with $|I| \geq \kappa$, and every $\kappa$-additive two-valued measure $\mu$ defined on some $B \subset \mathscr{P}(I)$, there is a $\kappa$-additive two-valued measure $\tilde{\mu} \supset \mu$ defined on all of $\mathscr{P}(I)$.

A cardinal $\kappa$ is real-valued measurable if $\kappa>\omega$ and there is a $\kappa$-additive measure defined on $\mathscr{P}(\kappa)$.

Solovay, $[10,4]$, has shown that if there is a model in which there is a $\kappa$ additive two-valued measure on $\mathscr{P}(I)$ then, by adding $\kappa$-many random reals, one obtains a model in which there is a $c$-additive real-valued measure on $\mathscr{P}(I)$. Therefore if $\kappa$ is a measurable cardinal and $\kappa$ random reals are added, there is a $c$-additive real-valued measurable cardinal (which is not measurable) in the resulting model. If $\kappa$ begins as a strongly compact cardinal then one obtains much more from Solovay's theorem. Indeed, in the ground model, there is a $\kappa$-additive two-valued measure on $\mathscr{P}(\lambda)$ for every $\lambda \geq \kappa$ (and we can make further demands on unif $(\mu)$ ). We are interested in formulating an axiom which will capture enough of the consequences of Solovay's results. Kunen has proven that there is a slightly different class of measures in this model and the associated axiom, PMEA, has become quite well known. Because of its popularity it seems more natural to use it as our basic axiom rather than to explicitly give a name to the above consequence of Solovay's theorem. The product measure extension 
axiom, PMEA, is said to hold if for every set $I$ there is a $\mathfrak{c}$-additive real-valued measure defined on $\mathscr{P}\left(2^{I}\right)$ which extends the usual product measure on $2^{I}$. We do not know if PMEA implies that, for each regular $\lambda \geq \mathfrak{c}$, there is a $\mathfrak{c}$ additive real-valued measure defined on $\mathscr{P}(\lambda)$ with unif $(\mu)=\lambda$. However we can easily observe from the proof as presented in [4], that the following holds in the model we are discussing.

Let PMEA $^{*}$ denote the statement: for every cardinal $\lambda \geq \mathfrak{c}$ there is a $\mathfrak{c}$ additive real-valued measure, $\mu$, defined on $\mathscr{P}\left(2^{\lambda}\right)$ and an embedding $\varphi$ of $[\lambda]^{<c}$ into $2^{\lambda}$ such that the following hold:

(1) $\mu$ extends the usual product measure on $2^{\lambda}$,

(2) $\mu\left(\varphi\left([\lambda]^{<\mathfrak{c}}\right)\right)=1$,

(3) for $a \in[\lambda]^{<c}$ and $\gamma \in \lambda \backslash a, \varphi(a)(\gamma)=0$.

We shall prove the following results in this section.

Theorem 4.1. If there is a real-valued measurable cardinal, $\kappa$, then $\mathfrak{C}$ does not equal TOP. In fact no space in $\mathfrak{C}$ witnesses tightness $\kappa$. Furthermore, if the measure is not two-valued then, there is a countable space which is not in $\mathfrak{C}$.

Theorem 4.2. If PMEA* holds then no space in $\mathfrak{C}$ witnesses tightness $\mathfrak{c}$ or larger.

The proof of the previous result can be easily simplified to prove the following.

Theorem 4.3. If there is a strongly compact cardinal $\kappa$, then no space in $\mathfrak{C}$ witnesses tightness $\kappa$ or larger.

To prove these results we formulate a topological property associated with a measure $\mu$.

Definition 4.4. Let $\mu$ be a measure defined on $\mathscr{P}(I)$. Let us say that a space $X$ is a $P(\mu)$-space (and has property $P(\mu)$ ), if, for every sequence $\mathscr{U}=\left\{U_{i}: i \in I\right\}$ of open subsets of $X$ and every real number $r$, the set

$$
\mathscr{U}_{r}=\left\{x \in X: \mu\left(\left\{i \in I: x \in U_{i}\right\}\right)>r\right\}
$$

is open in $X$.

Note that $\mathscr{U}_{1}=\varnothing$ and $\mathscr{U}_{-1}=X$. Also note that if $J \subset I$ is such that $\mu(J)>0$ we can define $\mu_{J}=\mu \mid \mathscr{P}(J)$. Then each $P(\mu)$-space is also a $P\left(\mu_{J}\right)$-space.

Lemma 4.5. Let $X$ be a space which witnesses tightness $\kappa$. If $\mu$ is a measure such that there are disjoint sets, $\left\{I_{\alpha}: \alpha<\kappa\right\}$, each of measure zero and whose union does not have measure zero, then $X$ is not a $P(\mu)$-space. In particular, if $\kappa \in\{\operatorname{add}(\mu), \operatorname{unif}(\mu)\}$, then $X$ is not a $P(\mu)$-space.

Proof. Suppose that $X$ is a space which witnesses tightness $\kappa$. Let $\left\{x_{\alpha}: \alpha<\right.$ $\kappa\} \subset X$ and $x \in X$ be such that $x \in \overline{\left\{x_{\beta}: \beta<\kappa\right\}}$ and $x \notin \overline{\left\{x_{\beta}: \beta<\alpha\right\}}$ for each $\alpha<\kappa$. Let $\mu$ be a measure on $\mathscr{P}(I)$. By our hypothesis on $\kappa$ we may fix pairwise disjoint sets, $\left\{I_{\alpha}: \alpha<\kappa\right\}$, of measure zero such that the union does not have measure zero (e.g. if $\kappa=\operatorname{unif}(\mu)$ then each $I_{i}$ is a singleton). For each $\alpha \in \kappa$ and $i \in I_{\alpha}$, let $U_{i}=X \backslash \overline{\left\{x_{\beta}: \beta<\alpha\right\}}$. For other values of $i$, let $U_{i}=\varnothing$. Define $\mathscr{U}=\left\{U_{i}: i \in I\right\}$. Clearly $x \in \mathscr{U}_{0}$, hence if $X$ were a $P(\mu)$-space, then the set $\mathscr{U}_{0} \cap\left\{x_{\beta}: \beta<\kappa\right\}$ would be nonempty. However, $\mu\left(\left\{i \in I: x_{\beta} \in U_{i}\right\}\right)=\mu\left(\bigcup_{\gamma \leq \beta} I_{\gamma}\right)=0$. Therefore $x_{\beta} \notin \mathscr{U}_{0}$. 
We have only a partial converse.

Lemma 4.6. If $X$ is not a $P(\mu)$-space, where $\mu$ is a two-valued measure, then $t(X) \geq \operatorname{add}(\mu)$.

Proof. Let $\mu$ be a measure on $I$ and fix a sequence $\mathscr{U}=\left\{U_{i}: i \in I\right\}$ of open subsets of $X$ which witness that $X$ is not a $P(\mu)$-space, i.e., $\mathscr{U}_{0}$ is not open. Choose a set $S \subset X \backslash \mathscr{U}_{0}$ of minimum cardinality such that $\bar{S} \cap \mathscr{U}_{0} \neq \varnothing$. For each $s \in S$, let $I_{s}=\left\{i \in I: s \in U_{i}\right\}$, hence $\mu\left(I_{s}\right)=0$. Suppose that $|S|<\operatorname{add}(\mu)$, hence $\mu\left(\bigcup_{s \in S} I_{s}\right)=0$. Let $x \in \mathscr{U}_{0} \cap \bar{S}$. Since $\mu\left(\left\{i \in I: x \in U_{i}\right\}\right)=1$, we may choose $i \in I \backslash \bigcup_{s \in S} I_{s}$ such that $x \in U_{i}$. Clearly $U_{i} \cap S=\varnothing$, contradicting that $x \notin \bar{S}$.

The following result shows that a direct converse of Lemma 4.5 does not hold. In the next section we raise related questions (see 5.5).

Lemma 4.7. If there is a real-valued measurable cardinal which is not two-valued measurable, then $\omega \cup\{p\}$ does not have property $P(\mu)$ for any $p \in \omega^{*}$.

Proof. Suppose that $\mu$ is a countably additive measure on $\mathscr{P}(I)$ which is not two-valued. We may assume that $\mu(I)=1$ and we have a measure preserving homomorphism from the usual measure space on $2^{\omega}$ into $\mathscr{P}(I) /\{A \subset I: \mu(A)=0\} \quad($ see $[5,7.3])$. Fix a measure independent family $\left\{I_{n}: n \in \omega\right\} \subset \mathscr{P}(I)$ so that $\mu\left(I_{n}\right)=\frac{1}{2}$ for each $n$. For each $i \in I$, let $U_{i}=\left\{n \in \omega: i \in I_{n}\right\} \cup\{p\}$ if this set is in $p$ otherwise let $U_{i}$ be $\{p\}$ union the complement. Let $\mathscr{U}=\left\{U_{i}: i \in I\right\}$ and let us show that $\mathscr{U}_{3 / 4}$ is finite and contains $p$. Let $S_{0}=\left\{i \in I: i \in I_{n}\right.$ for all $\left.n \in U_{i}\right\}$ and let $S_{1}=I \backslash S_{0}$. Since the family $\left\{I_{n}: n \in \omega\right\}$ is measure independent there are at most finitely many $n$ such that $\mu\left(I_{n} \cap S_{0}\right) \geq \frac{3}{4} \mu\left(S_{0}\right)$. Similarly, there are at most finitely many $n$ such that $\mu\left(S_{1} \backslash I_{n}\right) \geq \frac{3}{4} \mu\left(S_{1}\right)$. Now if $n$ is not one of these finitely many, then $\mu\left(\left\{i \in I: n \in U_{i}\right\}\right) \leq \mu\left(I_{n} \cap S_{0}\right)+\mu\left(S_{1} \backslash I_{n}\right)<\frac{3}{4}$.

The following is our motivation for defining the class of $P(\mu)$-spaces.

Lemma 4.8. If $\mu$ is a measure, then the class of $P(\mu)$-spaces is closed under finite products, arbitrary sums and quotients.

Proof. It is trivial that sums of $P(\mu)$-spaces are again $P(\mu)$-spaces. Suppose that $X$ is a $P(\mu)$-space and let $f: X \mapsto Y$ be a quotient map. Let $\mathscr{U}=$ $\left\{U_{i}: i \in I\right\}$ be open subsets of $Y$, where $\mu$ is a measure on $\mathscr{P}(I)$. Fix $r$ and let $\mathscr{U}_{r}=\left\{y \in Y: \mu\left(\left\{i \in I: y \in U_{i}\right\}\right)>r\right\}$. For each $i \in I$, let $W_{i}=f^{-1}\left(U_{i}\right)$ and $\mathscr{W}=\left\{W_{i}: i \in I\right\}$. Since $X$ is a $P(\mu)$-space, $\mathscr{W}_{r}$ is an open subset of $X$. To show that $\mathscr{U}_{r}$ is open, hence that $Y$ is a $P(\mu)$-space, it suffices to observe that $f^{-1}\left(\mathscr{U}_{r}\right)=\mathscr{W}_{r}$.

Now we turn to products. Suppose that $X$ and $Y$ are $P(\mu)$-spaces, we show that the product $X \times Y$ is also a $P(\mu)$-space. Fix $\mathscr{W}=\left\{W_{i}: i \in I\right\}$ a sequence of open subsets of $X \times Y$ and let $r \geq 0$. Suppose that $\left(x_{0}, y_{0}\right) \in \mathscr{W}_{r}$ and let $s=\mu\left(\left\{i \in I:\left(x_{0}, y_{0}\right) \in W_{i}\right\}\right)$. Fix $r^{\prime}>0$ with $r<r^{\prime}<s$ so that $s-2\left(s-r^{\prime}\right)>r$. For each $i \in I$ such that $\left(x_{0}, y_{0}\right) \in W_{i}$, choose open sets $U_{i} \subset X$ and $V_{i} \subset Y$ so that $\left(x_{0}, y_{0}\right) \in U_{i} \times V_{i} \subset W_{i}$. For other $i \in I$, let $U_{i}=V_{i}=\varnothing$. Since $X$ and $Y$ are $P(\mu)$-spaces, $\mathscr{U}_{r^{\prime}} \times \mathscr{V}_{r^{\prime}}$ is an open subset of $X \times Y$ which contains $\left(x_{0}, y_{0}\right)$, where $\mathscr{U}=\left\{U_{i}: i \in I\right\}$ and similarly for $\mathscr{V}$. 
Finally we check that $\mathscr{U}_{r^{\prime}} \times \mathscr{V}_{r^{\prime}} \subset \mathscr{W}_{r}$. Indeed, if $(x, y) \in \mathscr{U}_{r^{\prime}} \times \mathscr{V}_{r^{\prime}}$, then

$$
\begin{aligned}
\left\{i \in I:(x, y) \in W_{i}\right\} & \supset\left\{i \in I: x \in U_{i} \text { or } y \in V_{i}\right\} \\
& \supset\left(\left\{i \in I:\left(x_{0}, y_{0}\right) \in W_{i}\right\} \backslash\left\{i \in I: x \notin U_{i} \text { or } y \notin V_{i}\right\}\right) .
\end{aligned}
$$

Now this last set has measure greater than $s-2\left(s-r^{\prime}\right)>r$, hence $(x, y) \in$ $\mathscr{W}_{r}$.

It is curious to note that the above result does not require that the measure be countably additive. However the following immediate consequence of Lemmas 4.5 and 4.6 does.

Corollary 4.9. The converging sequence, $\omega+1$, is a $P(\mu)$-space if and only if the measure $\mu$ is countably additive.

We may collect the above two results in the following theorem.

Theorem 4.10. If $\mu$ is a countably additive measure, then every member of $\mathfrak{C}$ is a $P(\mu)$-space.

Proof of Theorem 4.1. Suppose that $\mu$ is a countably additive measure on $\mathscr{P}(I)$. By Theorem 4.10 , we have that every space in $\mathfrak{C}$ is a $P(\mu)$-space. Naturally, by Lemma 4.5 , we have that the space $\kappa \oplus 1$ is not a $P(\mu)$-space, where $\kappa=\operatorname{add}(\mu)$. The last statement in the theorem is proven by Lemma 4.7.

Proof of Theorem 4.2. Suppose that $X$ is a space and suppose that $\lambda \geq \mathfrak{c}$. Fix $x \in X$ and $\left\{x_{\beta}: \beta<\lambda\right\} \subset X$ such that $x \notin \overline{\left\{x_{\beta}: \beta \in a\right\}}$ for any $a \in[\lambda]^{<c}$. Let $\mu$ be a c-additive measure on $2^{\lambda}$ which extends the usual product measure and let $\varphi$ be an embedding of $[\lambda]^{<c}$ into $2^{\lambda}$ as in the statement of PMEA $^{*}$. For each $a \in[\lambda]^{<c}$, let $U_{\varphi(a)}$ be an open neighborhood of $x$ which is disjoint from $\left\{x_{\beta}: \beta \in a\right\}$. For $f \in 2^{\lambda} \backslash \varphi\left([\lambda]^{<c}\right)$, let $U_{f}=\varnothing$. For each $\beta \in \lambda$, the set $\left\{f \in 2^{\lambda}: x_{\beta} \in U_{f}\right\}$ is a subset of the set $\{\varphi(a): \beta \in a\}$. Therefore, by hypothesis 3 on $\varphi$, this set has measure at most $\frac{1}{2}$.

Now let us suppose that $X$ is a $P(\mu)$-space and let $\mathscr{U}=\left\{U_{f}: f \in 2^{\lambda}\right\}$. Therefore $\mathscr{U}_{1 / 2}$ is a neighborhood of $x$ which is disjoint from $\left\{x_{\beta}: \beta<\lambda\right\}$. This shows that $X$ does not witness tightness greater than or equal to $\mathfrak{c}$. By Theorem 4.10, this completes the proof. Just to further illustrate the axiom, let us remark that $\mathscr{U}_{0} \cap\left\{x_{\beta}: \beta<\lambda\right\}$ is finite.

Let us end the section by making the exact references to [4] to justify the claim that PMEA $^{*}$ holds in the Solovay model. We assume that reader has a copy of [4] on hand and we indicate exactly which observations must be made to justify the claim. In the proof of $[4,3.4]$, we may assume that $\left\{g \in 2^{\kappa}: g(0)=0\right\} \in G$ and that the set $I=[\lambda]^{<\kappa}$. The measure $\sigma$ of $[4,3.4]$ is the measure $\mu$ we seek subject to the restrictions that follow. The measure $\nu$ on $I$ is obtained from the $\kappa$-complete ultrafilter $\mathfrak{U}$ on $I$, from a ground model in which $\kappa$ is strongly compact, by applying $[4,3.3]$. The functions $f_{\alpha}:[\lambda]^{<\kappa} \mapsto \kappa$ are defined in the proof of $[4,3.1]$. The only hypothesis on $\mathfrak{U}$ from $[4,3.1]$ is that, for each $\alpha<\lambda$, the set $X_{\alpha}=\{a \in I: \alpha \in a\}$ is in $\mathfrak{U}$. Working in that ground model in which $\kappa$ is strongly compact, we may suppose that $\mathfrak{U}$ extends the $\kappa$-complete filter generated by

$$
\left\{X_{\alpha}: \alpha \in \lambda\right\} \cup\left\{I \backslash Y:|Y|<\left|[\lambda]^{<\kappa}\right|\right\} .
$$


Now $\nu$ has the property that $\nu(U)=1$ for all $U \in \mathfrak{U}$. Let $\varphi$ be as given in $[4,3.4]$; hence by definifion

$$
\sigma(\varphi(I))=\nu\left(\varphi^{-1}(\varphi(I))\right)=1 .
$$

It remains to show, in the present notation, that $\varphi\left(X_{\gamma}\right) \subset\left\{f \in 2^{\lambda}: f(\gamma)=0\right\}$. We wish to observe in $[4,3.1]$ that, for $\gamma \notin a \in[\lambda]^{<\kappa}, f_{\gamma}(a)=0$. Let $a \in$ $I \backslash X_{\gamma}$, we must show that $\varphi(a)(\gamma)=0$. Now $\varphi(a)(\gamma)=\varphi_{\gamma}(a)$, and $\varphi_{\gamma}(a)=1$ if and only if $\left\{g \in 2^{\kappa}: g\left(f_{\gamma}(a)\right)=1\right\} \in G$. Equivalently, $\varphi_{\gamma}(a)=0$ if and only if $\left\{g \in 2^{\kappa}: g\left(f_{\gamma}(a)\right)=0\right\} \in G$. Since $f_{\gamma}(a)=0$ and, we are assuming, without loss of generality, that $\left\{g \in 2^{\kappa}: g(0)=0\right\} \in G$, we have that $\varphi(a)(\gamma)=0$ as desired.

\section{QUESTIONS}

Of course, the main open question is the status of Husek's original question in ZFC. Given our results of $\S 2$ it is reasonable to pose the following question.

Question 5.1. If $\theta$ is a strongly compact cardinal, is the class of spaces which do not witness tightness precisely $\kappa$ for any $\kappa \geq \theta$ closed under products? Let us note that this class is closed under quotients.

Question 5.2. If $\theta$ is the minimal weakly compact cardinal (assuming it exists), does it follow that $\theta \oplus 1$ is not in $\mathfrak{C}$ ?

Discussion. It is certainly consistent that $\theta \oplus 1$ is not in $\mathfrak{C}$ (assuming that it is consistent to have a strongly compact cardinal with a weakly compact cardinal above it). Indeed, in any model in which $\mathrm{PMEA}^{*}$ holds and in which there is a weakly compact cardinal $\theta$, then $\theta \oplus 1$ will not be in $\mathfrak{C}$. However let us also note that it is known that there are models in which there are measurable cardinals and $\square_{\lambda}$ holds for arbitrarily large cardinals $\lambda$. Therefore, there are models in which there are cardinals $\theta$ such that $\theta \oplus 1 \notin \mathfrak{C}$ and yet for all regular cardinals $\lambda>\theta, \lambda \oplus 1 \in \mathfrak{C}$.

The above question is in a sense simply a special case of a more general problem of determining what effect quotients can have on tightness. It is of course known that they do not raise tightness (see $[3,3.12 .8]$ ) and it is easy to find examples where they lower tightness. However the following seems to be a good formulation of the problem.

Question 5.3. For a cardinal $\lambda$, let $\mathfrak{C}_{\lambda}$ denote the set obtained by relativizing the construction of $\mathfrak{C}$ to $H(\lambda)$-the set of sets whose transitive closure has cardinality less than $\lambda$ (possibly $V_{\lambda}$ may be more appropriate). Then if there is a space $X \in \mathfrak{C}$ which witnesses tightness $\lambda$ does it follow that there is a space in $\mathfrak{C}_{\lambda^{+}}\left(\right.$or in $\left.\mathfrak{C}_{\lambda+\omega}\right)$ which witnesses tightness $\lambda$ ? Does the space $\left(F_{\lambda, \mu}\right)^{2}$ witness tightness $\lambda$ ?

Discussion. If $\lambda$ is weakly compact, then there is no space in $\mathfrak{C}_{\lambda^{+}}$which witnesses tightness $\lambda$. Todorcevic [12] has shown that if $\lambda$ is less than the first weakly inaccessible cardinal, then there is a space in $\mathfrak{C}$ which witnesses tightness $\lambda$.

One may notice that in $\S \S 2$ and 3 we are always discussing the concept of witnessing tightness precisely $\theta$ and doing so with isolated points. Whereas in $\S 4$ all our results are about the simpler concept of witnessing tightness $\theta$ with no assumption about isolated points. 
Question 5.4. If there is a space $X$ in $\mathfrak{C}$ which witnesses tightness $\theta$, then is there a space in $\mathfrak{C}$ which witnesses tightness precisely $\theta$ ? If there is a space which witnesses tightness precisely $\theta$ in $\mathfrak{C}$, then is the space $\theta \oplus 1$ in $\mathfrak{C}$ ?

Another natural direction is to investigate further the relationship between $\mathfrak{C}$ and the $P(\mu)$-spaces.

Question 5.5. For a space $X$, let $\mathfrak{C}(X)$ denote the smallest subclass of TOP which contains $X$ and is closed under finite products, quotients and arbitrary sums, i.e. $\mathfrak{C}=\mathfrak{C}(\omega \oplus 1)$. If $X$ fails to have property $P(\mu)$ for some measure $\mu$, does it follow that there is a cardinal $\lambda$ (or a cardinal function on $\mu$ ) such that there is a space $Y$ in $\mathfrak{C}(X)$ such that $Y$ witnesses tightness $\lambda$ but no space in $\mathfrak{C}$ witnesses tightness $\lambda$ ?

Question 5.6. Does there exist $X \in \mathfrak{C}(\omega \cup\{p\})$ with tightness $\mathfrak{c}$, for each $p \in \omega^{*}$ ?

A very obvious problem is to determine the exact consistency strength of $\mathfrak{C} \neq$ TOP. Of course it has been established in this article that the relative consistency lies somewhere between the consistency of a weakly compact cardinal and a measurable cardinal. A more specific question is to ask if it involves measures.

Question 5.7. Does $\mathfrak{C} \neq$ TOP imply there is a real-valued measurable cardinal?

It would be very interesting to find a topological property which characterizes the class $\mathfrak{C}$.

Question 5.8. Does $\mathfrak{C}=\bigcap\{P(\mu): \mu$ is a countably additive real-valued measure\}?

\section{REFERENCES}

1. A. V. Arhangelskii, The spectrum of frequencies of a topological space and the classifications of spaces, Dokl. Akad. Nauk SSSR 206 (1972), 265-268.

2. Alan Dow, Istvan Juhasz, and William Weiss, Integer-valued functions and increasing unions of first countable spaces, Israel J. Math. 67 (1989), 181-192.

3. R. Engelking, General topology, PWN-Polish Scientific Publishers, 1977.

4. W. G. Fleissner, The normal Moore space conjecture and large cardinals, Handbook of Set Theoretic Topology, North-Holland, 1984, pp. 733-760.

5. David H. Fremlin, Measure algebras, Handbook of Boolean Algebras, vol. 3, North-Holland, 1989, pp. 877-980.

6. Horst Herrlich, Categorical topology 1971-1981, General Topology and its Relations to Modern Analysis and Algebra (J. Novak, ed.), Heldermann-Verlag, Belin, 1982, pp. 279383.

7. M. Husek, Special classes of compact spaces, Proceedings of the Berlin Conference on Categorical Topology (Berlin, 1978), Lecture Notes in Math., vol. 719, Springer-Verlag, 1979.

8. R. B. Jensen, The fine structure of the constructible hierarchy, Ann. of Math. Logic 4 (1972), 229-308.

9. V. I. Malyhin, On the tightness and the Suslin number of $\exp X$ and of a product of spaces, Soviet Math. Dokl. 4 (1972), 496-499.

10. R. M. Solovay, Real-valued measurable cardinals, Proc. Sympos. Pure Math., vol. 13, part 1, Amer. Math. Soc., Providence, R.I., 1971, pp. 397-428.

11. A. H. Stone, Metrizability of decomposition spaces, Proc. Amer. Math. Soc. 7 (1956), 690700 .

12. S. Todorcevic, My fans, May 1989, preprint. 
13. __ My new fan, May 1989, preprint.

14. __ Partitioning pairs of countable ordinals, Acta Math. 159 (1987), 261-294.

Department of Mathematics, York University, North York, Canada

E-mail address: dowa@nexus.yorku.ca

E-mail address: watson@vm1.yorku.ca 